\title{
Nasotracheal intubation with airway scope
}

\author{
Rui Ping Li $\cdot$ Fu Shan Xue $\cdot$ Shi Yu Wang
}

Received: 17 February 2014 / Accepted: 22 February 2014/Published online: 12 August 2014

(C) Japanese Society of Anesthesiologists 2014

\section{Keywords Airway management $\cdot$ Tracheal intubation}

\section{To the Editor:}

In a study assessing the performance of the airway scope (AWS) for nasotracheal intubation (NTI), Ono et al. [1] reported $100 \%$ success with cuff inflation among patients undergoing dental and maxillofacial surgery. They should be congratulated for their efforts in extending use of the AWS in airway management. However, several aspects of this study must be clarified. We believe such information would be useful for others who would like to try NTI with the AWS.

First, the design of the AWS used for orotracheal intubation. One of the most important features of the AWS, which facilitates intubation, is a target mark on the monitor. Before advancing the oral tube, the glottis must be positioned at the centre of the target mark. We would like to know whether the same relative positioning of the target mark and the glottis is required for successful NTI.

Second, the AWS blade is designed to be inserted posterior to the epiglottis, directly elevating it out of the way. In this study the AWS blade tip was positioned in the vallecula or epiglottis. The detailed reasons for the two blade positions were unclear. Did they affect the laryngoscopic view and subsequent NTI?

Third, cuff inflation was used as an aid to NTI with the AWS, but the authors did not specify in which situation this technique was used. In our experience, cuff inflation is effective only when the tube tip is excessively posterior or lateral to the glottis [2]. However, it is ineffective for anterior placement of the tube tip, which must be corrected by other means.

Conflict of interest None declared.

\section{References}

1. Ono $\mathrm{K}$, Goto $\mathrm{T}$, Nakai D, Ueki $\mathrm{S}$, Takenaka $\mathrm{S}$, Moriya $\mathrm{T}$. Incidence and predictors of difficult nasotracheal intubation with airway scope. J Anesth. 2014 Jan 17.

2. Xue FS, Lin JH, Liao X, Yuan YJ. Use of cuff inflation to facilitate nasotracheal intubation with the airway scope. Anaesthesia. 2011;66:754.
R. P. Li · F. S. Xue $(\bowtie) \cdot$ S. Y. Wang

Department of Anesthesiology, Plastic Surgery Hospital,

Chinese Academy of Medical Sciences, Peking Union Medical

College, 33 Ba-Da-Chu Road, Shi-Jing-Shan District,

Beijing 100144, People's Republic of China

e-mail: xuefushan@aliyun.com; fushan.xue@gmail.com 